\title{
1 Sexual deprivation modulates social interaction and reproductive physiology
}

2

3 Liora Omesi ${ }^{1}$, Mali Levi ${ }^{1}$, Assa Bentzur ${ }^{1}$, Yong-Kyu Kim²a, Shir Ben-Shaanan ${ }^{1}$, Reza Azanchi ${ }^{2 b}$, Ulrike

4 Heberlein $^{2}$ and Galit Shohat-Ophir ${ }^{1,3}$

5

6

$7 \quad{ }^{1}$ The Mina \& Everard Goodman Faculty of Life Sciences, The Leslie and Susan Gonda Multidisciplinary

8 Brain Research Center and The Nanotechnology Institute, Bar-Ilan University, Ramat Gan 5290002

9 Israel

10

11

${ }^{2}$ Janelia Research Campus, Howard Hughes Medical Institute, Ashburn, VA 20147, USA

12

13

14

15

16

$17 \quad{ }^{3}$ Lead contact

18

19 Correspondence should be addressed to G.S.O. (galit.ophir@biu.ac.il) 
Abstract

In highly polyandrous species, where females mate with multiple males within a single fertility

period, there is typically a high level of sperm competition. To cope with this challenge, males apply

various behavioral and physiological strategies to maximize their paternity rates. Previous studies in

Drosophila melanogaster established a link between the composition of the social environment and the

reproductive success of individual male flies. While most studies until now focused on the adaptive

responses of male flies to the presence of rival males, little is known about whether the outcomes of

sexual interactions with female partners affect male-male social interactions in a competitive

environment such as the social group. Here we show that repeated failures to mate promote a coordinated

physiological and behavioral responses that can serve to increase paternity chances over mating rivals.

We exposed male flies to sexual deprivation or successful mating and analyzed the behavioral repertoires

of individuals within groups and the structure of their emerging social networks. We discovered that

failures to mate and successful mating generate distinct emergent group interactions and structures,

where sexually deprived males form low density social networks and actively minimize their encounters

with other group members, while increasing their aggressive behavior. In addition, sexually deprived

male flies elevate the production of seminal fluid proteins (known to facilitate post-mating responses in

re-mating rates. Our results demonstrate the existence of a flexible mating strategy that may provide a

to dissect the neurobiology of social plasticity as coping strategy to living in a highly dynamic environment as the social domain. 


\section{Introduction}

The ability to adapt to environmental changes is an essential feature of biological systems, achieved in multicellular organisms by a coordinated crosstalk between neuronal and hormonal programs that generate plastic physiological and behavioral responses to environmental challenges ${ }^{1,2}$. This is particularly important in a dynamic, ever-changing and unpredictable environment, such as the social domain composed of many behaving animals, the interaction with ultimately determines the reproductive success of individuals ${ }^{2-4}$. The intricate nature of social interaction requires the ability to recognize other members of the group in the right context, season, sex, age and reproductive state, and integrate this information with prior experience to produce the appropriate and optimal behavioral response ${ }^{4}$. Plastic social responses are seen in diverse animals, and include modulation of competitive sexual behaviors such as mating preferences and aggressive displays, and also the regulation of social foraging and parental care $^{5-7}$. A remarkable example of social plasticity is evident in the African cichlid fish Astatotilapia burtoni, which live in a highly complex social environment consisting of many rival males that compete over limited food, territorial resources and female partners. Such a complex biotic and social environment produces a small number of dominant male fish and a large number of submissive males that closely monitor the social landscape in a constant search for opportunities to improve their social status, taking over mating territories and females ${ }^{7}$.

As a species with sociable lifestyle, Drosophila melanogaster exhibit communal living around freshly decaying fruits ${ }^{8}$ and engage in diverse forms of social interactions ${ }^{9}$. This includes courtship and mating $^{10,11}$, fighting over resources ${ }^{12}$, group interactions ${ }^{13}$, coordinated responses to threats ${ }^{14-16}$, cultural transmission of complex behaviors ${ }^{17}$, learning from conspecifics ${ }^{18,19}$, and synchronization of activity by social cues $^{20}$. Although some of these behaviors are considered innate responses, there are striking examples of the ability of fruit flies to exhibit social plasticity as they modulate their behavior and 
67 physiology in response to changes in their social environment. This includes the ability of male flies to

68 change their aggressive behavior in response to prior fighting experience ${ }^{21-23}$, regulate sperm

69 composition and the duration of copulation events in response to perceived competition ${ }^{24-26}$, and suppress

70 courtship efforts towards non-receptive female flies ${ }^{27-29}$.

71 Recent studies in Drosophila demonstrate that fruit flies generate complex and rich group

72 structures that are sensitive to the density of the group, its composition, as well as to the prior experience

73 of its members ${ }^{30-33}$. We previously showed that sexual experience in male flies can modulate their

74 motivational state and, subsequently, their reward seeking behaviors ${ }^{34,35}$. However less is known about

75 the way by which prior sexual interactions that are experienced as success or failure to mate shape social

76 interaction of male flies in a group context. Furthermore, it is not clear whether sexually deprived male

77 flies exhibit loser-like responses, as in the case of social defeat ${ }^{23}$, or rather actively increase their

78 competitive behavior to cope with mating rivals. Here we explored the effects of success or failure to

79 mate on the dynamics of social interaction in groups of male flies. We discovered that sexual deprivation

80 and successful mating generate opposite emergent group interactions and structures, wherein sexually

81 deprived male flies actively minimize their interactions with group members. Moreover, sexual

82 deprivation enhances competitive behaviors and leads to changes in reproductive physiology, possibly

83 to increase paternity chances over mating rivals. 
Results

\section{Failure to mate modifies action selection upon encounters with rival male flies}

We previously demonstrated that sexual experiences associated with different levels of mating success, such as repeated events of successful mating, or sexual deprivation in the form of repeated rejection events by non-receptive female flies, alter internal state and consequently motivational responses $^{34,35}$. The negative valence of rejection, reflected by its capacity to induce courtship suppression and increase the consumption of ethanol, prompted us to ask whether sexually deprived male flies exhibit loser-like responses ${ }^{23}$ or rather actively increase their competitive behavior to cope with mating rivals. To this end, we generated two cohorts of male flies that were exposed to repeated encounters with either receptive virgin female flies (mated-isolated) or non-receptive female flies (rejected-isolated), consisting of $1 \mathrm{~h}$ sessions 3 times a day for 4 days (Fig. 1A). At the end of this experience, their interactions in group context were tested by introducing 10 flies from each cohort into a shallow arena in which they could move and interact in two dimensions. Their behavior was recorded for $30 \mathrm{~min}$ and analyzed using the FlyBowl suite of tracking and behavior analysis softwares ${ }^{32,36,37}$ (Fig. 1A). The tracking data obtained was used to generate a comprehensive behavioral representation for each cohort composed of 60 distinct features, including kinetic features, eight distinct complex behaviors, and six social network features $(\text { Table } 1)^{32}$. The overall differences between the two cohorts across all features are depicted in a scatter plot of normalized differences and are divided into 4 main categories: activity-related features, interaction-related features, coordination between individuals, and features associated with social clustering (Fig. 1B). The two cohorts of male flies exhibited distinct repertoires of behavioral responses upon first encounters with other male flies. Sexually deprived male flies exhibited increased activity manifested as longer overall time spent walking, increased average velocity, and higher number of bodyturns (Fig. 1B, highlighted in pink, Supp Fig. 1A-C). When analyzing social-related behaviors, rejected male flies exhibited lower rates of close touch encounters (Fig. 1B, highlighted in blue, Supp Figure 1D), 
and while they displayed similar levels of active approaches towards other members of the group, the duration of these encounters was significantly shorter (Fig. 1B, highlighted in blue, Supp. Fig. 1E,F). In contrast, mated males exhibited long periods of quiescence (Fig. 2B, highlighted in blue, Supp. Fig. 1B),

111 and formed close-distance social (Fig. 1B, highlighted in blue, Supp Fig. 1G), reflected also by an

112 increase in the number of flies found in close proximity to one another (Fig. 1C).

\section{Failure to mate promotes social avoidance}

We next analyzed the properties of emerging social networks in both groups using weighted networks as described by Bentzur el al., ${ }^{32}$ (Fig. 2A). We calculated network weights according to the overall duration of interactions (emphasizing long-lasting interactions) or the overall number of interactions (emphasizing short interactions) between each pair of flies. Analysis by duration revealed that social networks of rejected males are characterized by lower density (Fig. 2B), reduced modularity (Fig. 2C), and reduced variation in individual strength levels across the group (SD strength, Fig. 2D).

These findings suggest that rejection promotes the formation of sparser groups containing fewer subgroups and that individuals in those groups are more homogenous in the strength of their interactions. Analysis by number of interactions revealed that, although rejected networks have lower modularity and $\mathrm{SD}$ strength, there is no significant differences in the density of their networks, suggesting that they maintain an overall similar number of interactions as mated male flies (Fig. 2E-G). Together, these differences indicate that mated male flies form networks with higher-order structures compared to those formed by rejected male flies. Notably, although rejected male flies participate in a similar number of interactions, their networks are simpler and sparser. The apparent differences in the density of networks measured by duration are consistent with significant differences between the two cohorts in the average distance between the two closest flies in each frame (dcenter), which is considerably higher in rejected males (Fig. 2H). More importantly, while in mated males the average distance between flies decreased 
132 flies (Fig. 2H). Considering that the elevated activity of rejected male flies (Fig. 1B) is expected to

133 increase the opportunity to encounter others, the maintenance of a larger distance throughout the

134 experiment and the reduced density suggest that rejected individuals actively avoid social interactions

135 with other flies. Together, these experiments point to sexual deprivation as the major contributor to the

136 reduced social interaction. To further test the strength of this conclusion, we divided a cohort of rejected-

137 isolated males into two subgroups, one of which was left undisturbed, and the other subgroup was

138 allowed to mate with virgin females for 2.5 hours immediately before testing. The rejected, then mated

139 sub-group exhibited intermediate levels of activity related features such as walk, stop, turn and average

140 velocity when compared to subgroups that had only experienced rejection or successful mating (Fig. 2I).

141 The rejected and then mated subgroup exhibited also intermediate degrees of social interaction related

142 features such as social clustering, number of flies found in close proximity to one another, and the levels

143 of grooming behavior that is tightly associated with social clustering (Fig. 2I). The capacity of mating to

144 partially reverse the effects of sexual deprivation is consistent with sexual deprivation being the major

145 contributor to social avoidance.

\section{Sexual deprivation modulates competitive behaviors}

Considering the major differences in group behavior displayed by rejected and mated male flies,

148 we hypothesized that the responses exhibited by rejected males reflect behavioral adaptation to coping

149 with high sexual competition over mating partners, where repeated encounters with mated females are

150 indicative of high male to female sex ratio. If so, rejected male flies are expected to increase behaviors

151 that provide them with an adaptive competitive value over rival male flies. This prediction can be tested

152 by measuring their aggressive responses toward other males in the presence of limited food resources or

153 their mating behavior upon opportunities to mate with virgin female flies. Indeed, pairs of rejected male

154 flies exhibited significantly higher aggressive displays in comparison to pairs of mated male flies (Fig.

$1553 \mathrm{~A}$ ), and that in mixed pairs, rejected males exhibited greater numbers of lunges compared to their mated 
counterparts (Fig. 3B,C). When allowed to mate with virgin female flies, rejected male flies extended

157 the duration of copulation events by $25 \%$ (3.5 minutes longer) compared to naïve males (Fig. 3D). Thus,

158 rejected male flies exhibited an overall increase in behaviors that can provide them with an adaptive

159 competitive value over rival male flies.

\section{Failure to mate induce changes in sperm and seminal fluid composition}

The act of mating alone does not guarantee fitness benefits including known strategies that reflect

male investment in sperm and non-sperm components, such as fecundity-enhancing seminal fluid proteins $^{38,39,40}$. To determine whether prior rejection affects reproductive physiology in a manner that may improve mating competitiveness, expression levels of genes related to sperm production and reproduction were assessed. First, the expression of DON-JUAN (DJ), a protein that is specifically

expressed in mature male sperm cells ${ }^{32,33}$, was measured using a GFP-based reporter line in which a GFP sequence was inserted within the coding locus, so that the expression of GFP reflects the expression of the endogenous DJ protein. The reliability of the DJ-GFP reporter as a sensitive measure for changes in sperm production was first confirmed in male flies raised among a high number of rival males (5 flies for 4 days), compared to the flies that were housed in pairs (Supp Fig.2), social conditions known to affect the amount of mature sperm ${ }^{25,41}$ (Supp Fig.2). The relative levels of GFP were then measured in rejected and naïve male flies (Fig. 4A-B). Surprisingly, there was a twofold decrease in the levels of GFP in the rejected cohort compared to naïve males (with no prior sexual experience), suggesting that male flies decrease their investment in sperm allocation in response to sexual deprivation (Fig. 4A-B). Next, 175 the relative expression of the following reproductive related genes was directly assessed in fly abdomens 176 by qRT-PCR. We measured the expression of Sex-Peptide (Acp70A), Acp63, Acp53, Ovulin (Acp26Aa), 177 which are responsible for the females' long-term post-mating responses and fertility ${ }^{38}$. We also measured 178 the expression of genes encoding the Ejaculatory bulb protein (Ebp), which is responsible for the 
posterior mating plug formation at the end of mating ${ }^{42}$, don-juan $(\mathrm{dj})^{40}$, the corazonin $(\mathrm{Crz})$ neuropeptide,

180 which promotes sperm and seminal fluid ejaculation in males and its receptor Crz-receptor ${ }^{35,43}$, and

181 finally Esterase 6 (est-6), an enzyme that is transferred to females during copulation and presumably

182 functions to degrade the pheromone $\mathrm{cVA}^{44}$ (Fig. 4C). There was a two-fold increase in the levels of $A c p-$

$18370 A$ (Sex-Peptide) and Acp-63 in rejected male flies when compared to naïve males, suggesting that

184 rejected male flies increase their investment in the production of seminal fluid proteins that are

185 transferred to females flies during copulation (Fig. 4C). Nevertheless, in agreement with the observed reduction in DJ-GFP reporter levels, there was a drastic decrease in the transcript levels of don-Juan in rejected males. The transcript levels of Ebp, Est-6, Crz and its receptor were similar in both cohorts (Fig. 4C). Overall, these results suggest that rejected male flies respond to sexual deprivation by elevating seminal fluid protein transcript levels, presumably to maximize their fitness. In addition to proteins associated with the male reproductive system, levels of several genes expressed in the brain and antenna were also assessed. These included the neuropeptides $C r z$, Neuropeptide $F$ (npf) and its receptor (npfr), and two olfactory related genes associated with aggression (the Odorant binding protein $69 a^{45}$, and

194 deprived male flies ${ }^{34}$; we also observed a reduction in $n p f r$ (Fig. 4D). Interestingly, sexually deprived

195 male flies also exhibited reduced levels of Cyp6a20 in comparison to naïve male flies (Fig. 4D), consistent with their enhanced aggression (Fig. 3 A-C).

\section{Females that mate with rejected male flies exhibit reduced re-mating behavior}

The molecular changes associated with the rejected condition support our initial hypothesis that rejected male flies adjust their behavior and physiology to cope with high sexual competition. If this is correct, the changes in seminal fluid composition and the extended copulation are expected to provide rejected male flies with an advantage over rival male flies. To test this prediction, several aspects 
counting the number of eggs they laid after one mating event with either rejected or naïve male flies.

204 There was no significant difference in the number of eggs laid across five days between the two cohorts 205 (Fig. 5A). The lack of difference in the amount of progeny suggested that lower sperm investment in 206 rejected males (as reflected by reduced DJ levels) does not affect the total offspring number, meaning 207 that there is no link between sperm investment and the number of offspring.

Next, we tested whether the increase in sex-peptide could facilitate enhanced post-mating behavior (such as reduced receptivity) in females that mated with rejected male flies. Since the strongest post-mating response is observed $24 \mathrm{~h}$ post mating (data not shown), the proportion of female flies that re-mated with new male flies $24 \mathrm{~h}$ after they mated with either rejected or naïve male flies was measured. A significant reduction was documented in the re-mating rates of females that mated initially with rejected versus virgin male flies (Fig. 5B), suggesting that extended copulation time and increase in seminal fluid proteins can lead to a stronger reduction in female receptivity. aphrodisiac pheromones such as $\mathrm{cVA}^{28}$. The extended copulation observed in rejected male flies may 217 facilitate the transfer of larger amounts of $\mathrm{cVA}$ as a means to delay further courtship and copulation 218 events by female flies. As an indirect measure for possible changes in the amount of transferred cVA, 219 we analyzed the courtship behavior of male flies towards females that previously mated with either 220 rejected or naive male flies $1 \mathrm{~h}$ after the initial mating. No significant difference was observed in the latency to court, i.e. the time it takes male flies to exhibit their first courtship action (wing vibration) 222 following introduction of the pair into the courtship arena (Fig. 5C). However, there was a significant 223 reduction in the number of male flies that courted females previously mated with rejected males than 224 those previously mated with naïve male flies (Fig. 5D), suggesting that mating with rejected male flies 225 results in females that are less attractive courtship targets. 


\section{Discussion}

In this study we used the FlyBowl ${ }^{37}$ as an agnostic tool to explore responses modulated by sexual interaction and discovered that rejected male flies cope with their failures to mate by changing their behavior and physiology to enhance their reproductive success. This is presumably achieved by avoiding interaction with potential rival male flies and competing over mating partners via increased aggression and prolonged copulation; this is known as mate guarding. The latter is strengthened by the increased production of certain seminal fluid proteins that facilitate stronger post-mating responses in female flies.

The behavior of sexually deprived male flies was examined in this study under behavioral contexts that illuminate different aspects of their action selection. Using the FlyBowl system, we analyzed their emergent group interactions and social networks, and discovered that although rejected males are highly active, they exhibit sparse networks and maintain large distance with other members, as if they were actively minimizing or avoiding interaction with rival male flies. When tested in a social context that promotes fighting over limited resources, rejected male flies exhibited enhanced aggression. in the levels of Cyp6a20. This is consistent with a previous study showing that Cyp6a20 levels are reduced in social conditions that promote aggression and that this reduction is responsible for the observed increase in aggression ${ }^{46}$. Interestingly, exposure to female flies prior to male-male interactions was previously shown to suppress aggression ${ }^{47}$. However, our findings suggest that not all types of interactions with female flies are sufficient for suppressing aggression, but rather that the quality of the interactions (i.e., the male's sexual success) determines the resulting aggression levels when encountering another male fly.

There are two possible explanations for the behavioral responses exhibited by rejected male flies.

248 First, failure to mate could enhance aggression to improve the chances of successful mating and, upon 
eventual mating, the increased duration of copulation could increase the relative paternity share. Second,

repeated rejection experiences could be perceived by male flies as an indication for high density of sexual competition over mating partners, where encountering mated females is suggestive of high male to female sex ratio. Consistent with the second hypothesis, several studies have described a link between pre-exposure to rival male flies and an extension of copulation events ${ }^{24,48}$. One study also demonstrated that male flies use multiple sensory cues such as auditory, olfactory and gustatory signals to estimate the level of mating competition ${ }^{48}$. Although rejected males were not exposed directly to other male flies during the training phase, the observed extension of their copulation events suggests that they can assess the level of competition by evaluating the quality of their sexual interaction with female flies. Studies performed in Pieris rapae butterflies, in which virgin males were shown to allocate their sperm investment by assessing not only the mating status of the female, but also her previous mating history ${ }^{49}$, are consistent with this hypothesis.

The behavioral responses to sexual deprivation were accompanied by changes in the repertoire of genes expressed in the brain and reproductive system in the form of increased expression of several accessory gland protein genes (Acps). This, together with the increased copulation duration, supports the idea that the observed extension in mating duration serves to transfer a higher amount of Acps to intensify the females' post- mating responses ${ }^{23,79}$. Unlike previous studies that demonstrated a link between the presence of rival male flies and an increase in both copulation duration and sperm allocation (measured by increase number of sperm cells $)^{25}$, rejected male flies exhibited a significant reduction in the levels of DJ, a protein expressed in mature sperm cells. Although this finding is limited to only one protein, this is surprising in light of sperm competition theory, which predicts that males should strategically increase their investment in sperm allocation when in competition ${ }^{50}$. Furthermore, our findings are different from studies in crickets, sunfish, birds and rats, which showed that the perceived risk of sperm competition, in 
sperm investment ${ }^{49,51,52}$. The unexpected uncoupling between the investment in sperm and non-sperm

274 components and the regulation of investment in copulation time, demonstrates that sexually deprived 275 male flies regulate each of these processes independently.

Functionally, the observed decrease in sperm quantity with increasing seminal fluid protein (Acp) expression in rejected males did not affect the amount of progeny produced in females. This observation suggests that there is no link between the observed behavioral and physiological changes and the amount of progeny. Nevertheless, females that mated with rejected males were less attractive to naïve male flies, as reflected by the reduced number of male flies that courted these females. The combination of reduced 281 female attractiveness in subsequent mating encounters, and reduced motivation of the female to re-mate, 282 may reduce the odds for a second mating and thus increase the rejected male's paternity rate despite the 283 lack of an effect on progeny number. events of rejection that gives them a short-term advantage, promoting reproductive fitness when competing with rival male flies. We postulate that rejected males invest more energy in the production of seminal fluid proteins over sperm; these Acps are known to have important roles in modulating different aspects of female mating physiology and behavior. Furthermore, at low population density, the chances to meet a receptive female are low, therefore an investment in sperm ejaculate may be more $\operatorname{costly}^{53}$. Further research is needed to dissect the molecular and neuronal mechanisms that mediate these 291 adaptive responses, identify the sensory modalities that perceive failure to mate, which encode this 292 information within the nervous system leading to ejaculate plasticity. 
295 Acknowledgments: We thank all members of the Shohat-Ophir lab for fruitful discussions and technical 296 support. We specially thank Kristin Branson and Alice Robie (HHMI Janelia research campus) for their 297 valuable advice and guidance with the experimental and computational design. This work was supported 298 by the Israel Science Foundation grant 384/14 and Israel Science Foundation grant 174/19. 
Materials and methods:

$300 \quad$ Fly lines and culture

301 Canton S flies were used as the wild-type strain. Flies were raised at $25^{\circ} \mathrm{C}$ in a 12 -h light/12-h dark cycle 302 in 60\% relative humidity and maintained on cornmeal, yeast, molasses, and agar medium, and were tested 303 as 3-4-day old adults, unless otherwise specified. The DJ-GFP and White Berlin (WB) lines were 304 obtained from the HHMI Janelia Farm Research Campus.

\section{Sexual experience paradigm}

306 Male and female flies were anesthetized under CO2 and isolated immediately after eclosion. Flies were 307 reared as single-housed in vials $(23 \mathrm{~mm}$ by $94 \mathrm{~mm}$ ) containing $7 \mathrm{ml}$ of medium and were aged separately 308 for 3-4 day. Rejected and mated cohorts were generated as previously described ${ }^{34}$. In the naïve cohort, 309 male flies were isolated for 4 days.

311 Social group interaction using the FlyBowl system

312 At the end of the sexual experience phase, rejected and mated male flies were inserted in groups of 10 313 into Fly Bowl arenas ${ }^{36}$, and their behavior was recorded for 30 minutes and analyzed using CTRAX, 314 FixTrax $^{32}$ and JAABA ${ }^{36}$. For kinetic features, scripts were written in MATLAB to use the JAABA code 315 to generate the statistical features as specified in Kabra et al. ${ }^{36}$. Time series graphs (per frame) were 316 created using JAABA Plot ${ }^{36}$. Quantification of complex behavios was done using JAABA Classifiers ${ }^{36}$ 317 to identify specific behaviors: Walk, Stop, Turn, Approach, Touch, Chase, Chain, Song, Social 318 Clustering and Grooming. Bar graphs were created using JAABA Plot ${ }^{36}$. Network analysis was 319 performed using an interaction matrix according to the interaction parameters described previously ${ }^{32}$. 320 Two interaction matrices were created for each movie, one with the total number of frames each pair of 321 flies were interacting divided by the number of frames in the movie and another with the number of 
322

separate interactions between each pair of flies divided by the maximum number of possible interactions, calculated as:

$$
\text { max \# of interaction possible } \frac{\# \text { of frames }- \text { min \# of frames for interaction }}{\min \# \text { of frames for interaction }+ \text { min } \# \text { of gap frames }}+1
$$

The parameters to define an interaction are: angle subtended by the other fly $>0$, distance between the nose of current fly to any point on the other fly $\leq 8 \mathrm{~mm}$, number of frames for interaction $\geq 60$ and number of gap frames $\geq 120$. Interaction end is defined when distance or angle conditions are not maintained for 4 seconds. Networks and their features were generated from the interaction matrix in $\mathrm{R}$ using the igraph package. The function that was used to the generate networks is "graph_from_adjacency_matrix" with parameters "mode = undirected" and "weighted $=$ TRUE". Density was calculated on all movies with the formula:

Modularity was calculated using the "modularity" function on output from the "cluster_walktrap" function ${ }^{54}$. Strength was calculated using "strength" function and SD Strength was calculated on all movies using "sd" function on the strength value. Betweenness Centrality was calculated on all flies using the "betweenness" function and SD Betweenness Centrality was calculated on all movies using "sd" function on the Betweenness Centrality value. Box plots were created using R.

Each feature of the FlyBwol experiment was standardized according to all values calculated in our experiments for that feature to generate a z-score. Scatter plots were created using R. 
$\underline{\text { Aggression }}$

347 Pairs of rejected or mated male flies were introduced into aggression arenas (circular chambers, about $3480.08 \mathrm{~cm} 3$ in volume), which contained a mixture of agarose and apple juice (1\% agarose, $50 \%$ apple 349 juice) that was placed in arenas to enhance aggressive behavior. Flies were filmed for 30 min with Point350 Grey Flea3 $(1080 \times 720$ pixels $)$ at $60 \mathrm{fps}$. Aggressive behavior was later quantified by counting the number 351 of lunges for each pair using CADABRA software (ref). The $\log _{2}$ ratio between the number of lunges in 352 rejected and mated flies was calculated for each pair, and then a one-sample t-test was performed to test 353 whether the mean ratio is significantly different from 0 .

Copulation duration

Rejected and naïve male flies were put into courtship arenas (circular chambers, about $0.04 \mathrm{~cm} 3$ in volume) with virgin females and were allowed to mate for 1 hour. They were recorded for the whole experiment using a Point-Grey firefly camera. Courtship arenas consist of 25 flat arenas each arena containing only one pair of male-female flies. The copulation duration was measured from the moment the mating began until it ended. We calculated the time in seconds for each fly and the average for each group.

Egg laying assay

364 Egg production was determined for females that had been allowed to copulate with rejected or naive 365 males for 1 hour at the end of the conditioning (as described above). Every female was put in a glass vial containing fresh food every day for 5 days in total and was kept in the incubator. Days 3 and 4 have received approximated values since day 3 was Saturday and we couldn't replace the vail that day; therefore, we tried to divide the number of eggs equally. Eggs can be spotted easily as circular white dots on the surface of the medium. The sum of the number of eggs in the vials of each female was used for analysis. 
bioRxiv preprint doi: https://doi.org/10.1101/2021.04.27.441612; this version posted April 28, 2021. The copyright holder for this preprint (which

was not certified by peer review) is the author/funder, who has granted bioRxiv a license to display the preprint in perpetuity. It is made available under aCC-BY-NC-ND 4.0 International license.

371

\section{$\underline{\text { Receptivity assay }}$}

3-4-day old White Berlin (WB) females were allowed to mate once with rejected or naïve males at the end of the conditioning for $1 \mathrm{~h}$. After mating, the males were disposed and the mated females were kept in the incubator for 24h. Afterward, the mated females were exposed to 5-day-old WT naïve males for 1 $\mathrm{h}$ to measure their receptivity to mate. Approximately 40 pairs of each group (rejected or naive) were set up in every biological repeat.

\section{Latency to court assay}

1 hour after allowing WB females to mate with rejected or naïve males, we transferred the females into courtship arenas and paired them with new WT naïve males. The pairs were recorded for 15 min to measure courtship latency. Latency was defined as the time elapsing between the introduction of the pair into the chamber and the first appearance of wing vibration made by the courting male fly. We also quantified the number of males who did and did not try to court in this assay.

\section{Courtship Index}

Courtship index for a given male is the fraction of time a male fly spent in courtship activity in the 10 min observation period $(600 \mathrm{sec})$. It is calculated by dividing the number of seconds the male courted over the total observation time and is been exhibit in percentage $(\mathrm{CI}=$ courtship behavior $[\mathrm{sec}] \cdot 100 /$ total observation $[\mathrm{sec}])$.

\section{Molecular methods}

Western blot analysis: Sperm allocation in male flies carrying the DJ-GFP reporter was determined by Western blotting. DJ protein size is $\sim 29 \mathrm{kDa}$, and GFP size is $\sim 25 \mathrm{kDa}$. We also determined the levels of Sex-peptide (SP), a protein of size $\sim 7 \mathrm{kDa}$, and the levels of Tubulin for normalization. The primary antibodies used were mouse anti-GFP, rabbit anti-SP and rabbit anti-Tubulin, and the secondary 
antibodies that were used are rabbit $\alpha$-mouse HRP and mouse $\alpha$-rabbit HRP, respectively. Virgin females 397 were used as negative controls.

Quantitative Real-Time PCR analysis

400 Frozen flies were placed on ice and decapitated using a scalpel. Total RNA was extracted from $\sim 15$ 401 frozen heads and bodies (separately), using TRIZOL reagent according to the manufacturer's protocol. 402 mRNA was reverse transcribed using BIORAD cDNA synthesis kit. cDNA was analyzed by quantitative real-time PCR (BIORAD CFX96) using specific primers for the head and for the body. Relative expression was quantified by $\Delta \Delta \mathrm{CT}$ method using RPL $32^{55}$ as a loading control. We run each sample in triplicates. Each experiment was repeated four times using independent sets of experimental flies.

$\underline{\text { Statistical analysis }}$

For each experiment, Shapiro-Wilk test was done on each experiment to test for normal distribution. Statistical significance was determined by t-test for experiments that were distributed normally, and by Wilcoxon test for experiments that were not distributed normally. For experiments with three or four conditions: statistical significance determined by one-way ANOVA followed by Tukey's range test for experiments that were distributed normally, and by Kruskal-Wallis test followed by Wilcoxon signedrank test for experiments that were not distributed normally. 


\section{References}

1. Robinson, G.E., Fernald, R.D., and Clayton, D.F. (2008). Genes and Social Behavior. Science 322, 896-900.

2. Oliveira, R.F. (2009). Social behavior in context: Hormonal modulation of behavioral plasticity and social competence. Integr. Comp. Biol. 49, 423-440.

421

422

423

424

425

426

427

428

429

430

431

432

433

434

435

436

437

438

439

440

441

442

443

444

445

446

447

3. Cardoso, S.D., Teles, M.C., and Oliveira, R.F. (2015). Neurogenomic mechanisms of social plasticity. J. Exp. Biol. 218, 140-149.

4. Taborsky, B., and Oliveira, R.F. (2012). Social competence: an evolutionary approach. Trends Ecol. Evol. 27, 679-688.

5. Jennions, M.D., and Petrie, M. (1997). Variation in Mate Choice and Mating Preferences: A Review of Causes and Consequences. Biol. Rev. 72, 283-327.

6. O’Connell, L.A., and Hofmann, H.A. (2012). Evolution of a Vertebrate Social Decision-Making Network. Science 336, 1154-1157.

7. Fernald, R.D. (2017). Cognitive skills and the evolution of social systems. J. Exp. Biol. 220, $103-113$.

8. Soto-Yéber, L., Soto-Ortiz, J., Godoy, P., and Godoy-Herrera, R. (2018). The behavior of adult Drosophila in the wild. PloS One 13, e0209917.

9. Sokolowski, M.B. (2010). Social interactions in "simple” model systems. Neuron 65, 780-794.

10. Dickson, B.J. (2008). Wired for Sex: The Neurobiology of Drosophila Mating Decisions. Science 322, 904-909.

11. Anderson, D.J. (2016). Circuit modules linking internal states and social behaviour in flies and mice. Nat. Rev. Neurosci. 17, 692-704.

12. Wang, L., Han, X., Mehren, J., Hiroi, M., Billeter, J.-C., Miyamoto, T., Amrein, H., Levine, J.D., and Anderson, D.J. (2011). Hierarchical chemosensory regulation of male-male social interactions in Drosophila. Nat. Neurosci. 14, 757-762.

13. Ramdya, P., Schneider, J., and Levine, J.D. (2017). The neurogenetics of group behavior in Drosophila melanogaster. J. Exp. Biol. 220, 35-41.

14. Elliott, K.H., Betini, G.S., and Norris, D.R. (2017). Fear creates an Allee effect: experimental evidence from seasonal populations. Proc. Biol. Sci. 284.

15. Ramdya, P., Lichocki, P., Cruchet, S., Frisch, L., Tse, W., Floreano, D., and Benton, R. (2015). Mechanosensory interactions drive collective behaviour in Drosophila. Nature 519, 233-236.

16. Ferreira, C.H., and Moita, M.A. (2020). Behavioral and neuronal underpinnings of safety in numbers in fruit flies. Nat. Commun. 11, 4182. 
17. Danchin, E., Nöbel, S., Pocheville, A., Dagaeff, A.-C., Demay, L., Alphand, M., Ranty-Roby, S., van Renssen, L., Monier, M., Gazagne, E., et al. (2018). Cultural flies: Conformist social learning in fruitflies predicts long-lasting mate-choice traditions. Science 362, 1025-1030.

18. Kacsoh, B.Z., Bozler, J., Ramaswami, M., and Bosco, G. (2015). Social communication of predatorinduced changes in Drosophila behavior and germ line physiology. eLife 4.

19. Mery, F., Varela, S.A.M., Danchin, E., Blanchet, S., Parejo, D., Coolen, I., and Wagner, R.H. (2009). Public versus personal information for mate copying in an invertebrate. Curr. Biol. CB 19, 730-734.

20. Levine, J.D., Funes, P., Dowse, H.B., and Hall, J.C. (2002). Resetting the Circadian Clock by Social Experience in Drosophila melanogaster. Science 298, 2010-2012.

21. Yurkovic, A., Wang, O., Basu, A.C., and Kravitz, E.A. (2006). Learning and memory associated with aggression in Drosophila melanogaster. Proc. Natl. Acad. Sci. U. S. A. 103, 17519-17524.

22. Liu, W., Liang, X., Gong, J., Yang, Z., Zhang, Y.-H., Zhang, J.-X., and Rao, Y. (2011). Social regulation of aggression by pheromonal activation of Or65a olfactory neurons in Drosophila. Nat. Neurosci. 14, 896-902.

23. Kim, Y.-K., Saver, M., Simon, J., Kent, C.F., Shao, L., Eddison, M., Agrawal, P., Texada, M., Truman, J.W., and Heberlein, U. (2018). Repetitive aggressive encounters generate a long-lasting internal state in Drosophila melanogaster males. Proc. Natl. Acad. Sci. U. S. A. 115, 1099-1104.

24. Kim, W.J., Jan, L.Y., and Jan, Y.N. (2013). A PDF/NPF neuropeptide signaling circuitry of male Drosophila melanogaster controls rival-induced prolonged mating. Neuron 80, 1190-1205.

25. Garbaczewska, M., Billeter, J.-C., and Levine, J.D. (2013). Drosophila melanogaster males increase the number of sperm in their ejaculate when perceiving rival males. J. Insect Physiol. 59, 306-310.

26. Hopkins, B.R., Sepil, I., Thézénas, M.-L., Craig, J.F., Miller, T., Charles, P.D., Fischer, R., Kessler, B.M., Bretman, A., Pizzari, T., et al. (2019). Divergent allocation of sperm and the seminal proteome along a competition gradient in Drosophila melanogaster. Proc. Natl. Acad. Sci. U. S. A. 116, 1792517933.

27. Siegel, R.W., and Hall, J.C. (1979). Conditioned responses in courtship behavior of normal and mutant Drosophila. Proc. Natl. Acad. Sci. U. S. A. 76, 3430-3434.

28. Keleman, K., Vrontou, E., Krüttner, S., Yu, J.Y., Kurtovic-Kozaric, A., and Dickson, B.J. (2012). Dopamine neurons modulate pheromone responses in Drosophila courtship learning. Nature 489, $145-149$.

29. Ejima, A., Smith, B.P.C., Lucas, C., van der Goes van Naters, W., Miller, C.J., Carlson, J.R., Levine, J.D., and Griffith, L.C. (2007). Generalization of courtship learning in Drosophila is mediated by cisvaccenyl acetate. Curr. Biol. CB 17, 599-605.

30. Rooke, R., Rasool, A., Schneider, J., and Levine, J.D. (2020). Drosophila melanogaster behaviour changes in different social environments based on group size and density. Commun. Biol. 3, 304. 
31. Schneider, J., Dickinson, M.H., and Levine, J.D. (2012). Social structures depend on innate determinants and chemosensory processing in Drosophila. Proc. Natl. Acad. Sci. U. S. A. 109 Suppl 2, 17174-17179.

32. Bentzur, A., Ben-Shaanan, S., Benichou, J.I.C., Costi, E., Levi, M., Ilany, A., and Shohat-Ophir, G. (2020). Early Life Experience Shapes Male Behavior and Social Networks in Drosophila. Curr. Biol. CB.

33. Battesti, M., Pasquaretta, C., Moreno, C., Teseo, S., Joly, D., Klensch, E., Petit, O., Sueur, C., and Mery, F. (2015). Ecology of information: social transmission dynamics within groups of non-social insects. Proc. Biol. Sci. 282, 20142480.

34. Shohat-Ophir, G., Kaun, K.R., Azanchi, R., Mohammed, H., and Heberlein, U. (2012). Sexual deprivation increases ethanol intake in Drosophila. Science 335, 1351-1355.

35. Zer-Krispil, S., Zak, H., Shao, L., Ben-Shaanan, S., Tordjman, L., Bentzur, A., Shmueli, A., and Shohat-Ophir, G. (2018). Ejaculation Induced by the Activation of Crz Neurons Is Rewarding to Drosophila Males. Curr. Biol. CB 28, 1445-1452.e3.

36. Kabra, M., Robie, A.A., Rivera-Alba, M., Branson, S., and Branson, K. (2012). JAABA: interactive machine learning for automatic annotation of animal behavior. Nat. Methods 10, 64-67.

37. Branson, K., Robie, A.A., Bender, J., Perona, P., and Dickinson, M.H. (2009). High-throughput ethomics in large groups of Drosophila. Nat. Methods 6, 451-457.

38. Sirot, L.K., LaFlamme, B.A., Sitnik, J.L., Rubinstein, C.D., Avila, F.W., Chow, C.Y., and Wolfner, M.F. (2009). Molecular social interactions: Drosophila melanogaster seminal fluid proteins as a case study. Adv. Genet. 68, 23-56.

39. Wigby, S., Brown, N.C., Allen, S.E., Misra, S., Sitnik, J.L., Sepil, I., Clark, A.G., and Wolfner, M.F. (2020). The Drosophila seminal proteome and its role in postcopulatory sexual selection. Philos. Trans. R. Soc. B Biol. Sci. 375, 20200072.

40. Santel, A., Winhauer, T., Blümer, N., and Renkawitz-Pohl, R. (1997). The Drosophila don juan (dj) gene encodes a novel sperm specific protein component characterized by an unusual domain of a repetitive amino acid motif. Mech. Dev. 64, 19-30.

41. Moatt, J.P., Dytham, C., and Thom, M.D.F. (2014). Sperm production responds to perceived sperm competition risk in male Drosophila melanogaster. Physiol. Behav. 131, 111-114.

42. Avila, F.W., Cohen, A.B., Ameerudeen, F.S., Duneau, D., Suresh, S., Mattei, A.L., and Wolfner, M.F. (2015). Retention of Ejaculate by Drosophila melanogaster Females Requires the Male-Derived Mating Plug Protein PEBme. Genetics 200, 1171-1179.

43. Tayler, T.D., Pacheco, D.A., Hergarden, A.C., Murthy, M., and Anderson, D.J. (2012). A neuropeptide circuit that coordinates sperm transfer and copulation duration in Drosophila. Proc. Natl. Acad. Sci. U. S. A. 109, 20697-20702. 
44. Chertemps, T., François, A., Durand, N., Rosell, G., Dekker, T., Lucas, P., and Maïbèche-Coisne, M. (2012). A carboxylesterase, Esterase-6, modulates sensory physiological and behavioral response dynamics to pheromone in Drosophila. BMC Biol. 10, 56.

45. Bentzur, A., Shmueli, A., Omesi, L., Ryvkin, J., Knapp, J.-M., Parnas, M., Davis, F.P., and ShohatOphir, G. (2018). Odorant binding protein 69a connects social interaction to modulation of social responsiveness in Drosophila. PLoS Genet. 14, e1007328.

46. Wang, L., Dankert, H., Perona, P., and Anderson, D.J. (2008). A common genetic target for environmental and heritable influences on aggressiveness in Drosophila. Proc. Natl. Acad. Sci. U. S. A. $105,5657-5663$.

47. Yuan, Q., Song, Y., Yang, C.-H., Jan, L.Y., and Jan, Y.N. (2014). Female contact modulates male aggression via a sexually dimorphic GABAergic circuit in Drosophila. Nat. Neurosci. 17, 81-88.

48. Bretman, A., Westmancoat, J.D., Gage, M.J.G., and Chapman, T. (2011). Males use multiple, redundant cues to detect mating rivals. Curr. Biol. CB 21, 617-622.

49. Wedell, N., and Cook, P.A. (1999). Butterflies tailor their ejaculate in response to sperm competition risk and intensity. Proc. R. Soc. Lond. B Biol. Sci. 266, 1033-1039.

50. Parker, G.A., Ball, M.A., Stockley, P., and Gage, M.J. (1997). Sperm competition games: a prospective analysis of risk assessment. Proc. R. Soc. B Biol. Sci. 264, 1793-1802.

51. Gage, M.J.G. (1991). Risk of sperm competition directly affects ejaculate size in the Mediterranean fruit fly. Anim. Behav. 42, 1036-1037.

52. Nicholls, E.H., Burke, T., and Birkhead, T.R. (2001). Ejaculate Allocation by Male Sand Martins, Riparia riparia. Proc. Biol. Sci. 268, 1265-1270.

53. Parker, G.A., and Pizzari, T. (2010). Sperm competition and ejaculate economics. Biol. Rev. 85, 897-934.

54. Pons, P., and Latapy, M. (2005). Computing communities in large networks using random walks. In Lecture Notes in Computer Science (including subseries Lecture Notes in Artificial Intelligence and Lecture Notes in Bioinformatics) (Springer, Berlin, Heidelberg), pp. 284-293.

55. Zheng, Y.T., Li, H.B., Lu, M.X., and Du, Y.Z. (2014). Evaluation and validation of reference genes for qRT-PCR normalization in Frankliniella occidentalis (Thysanoptera: Thripidae). PLoS ONE 9. 
A. O'
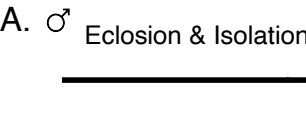

Day 1
Rejection

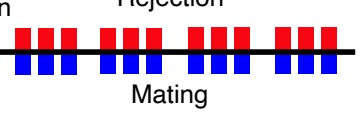

$5 \quad 6$

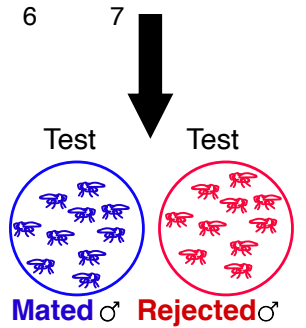

C.

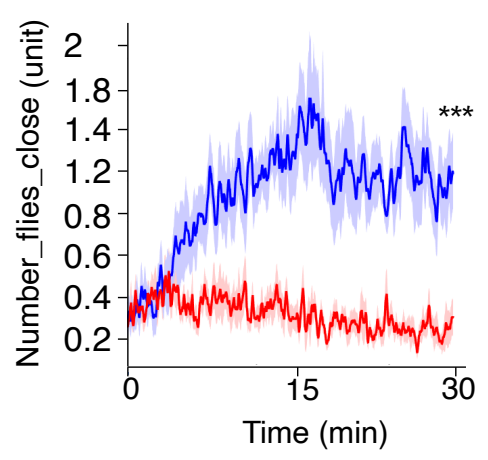

B.

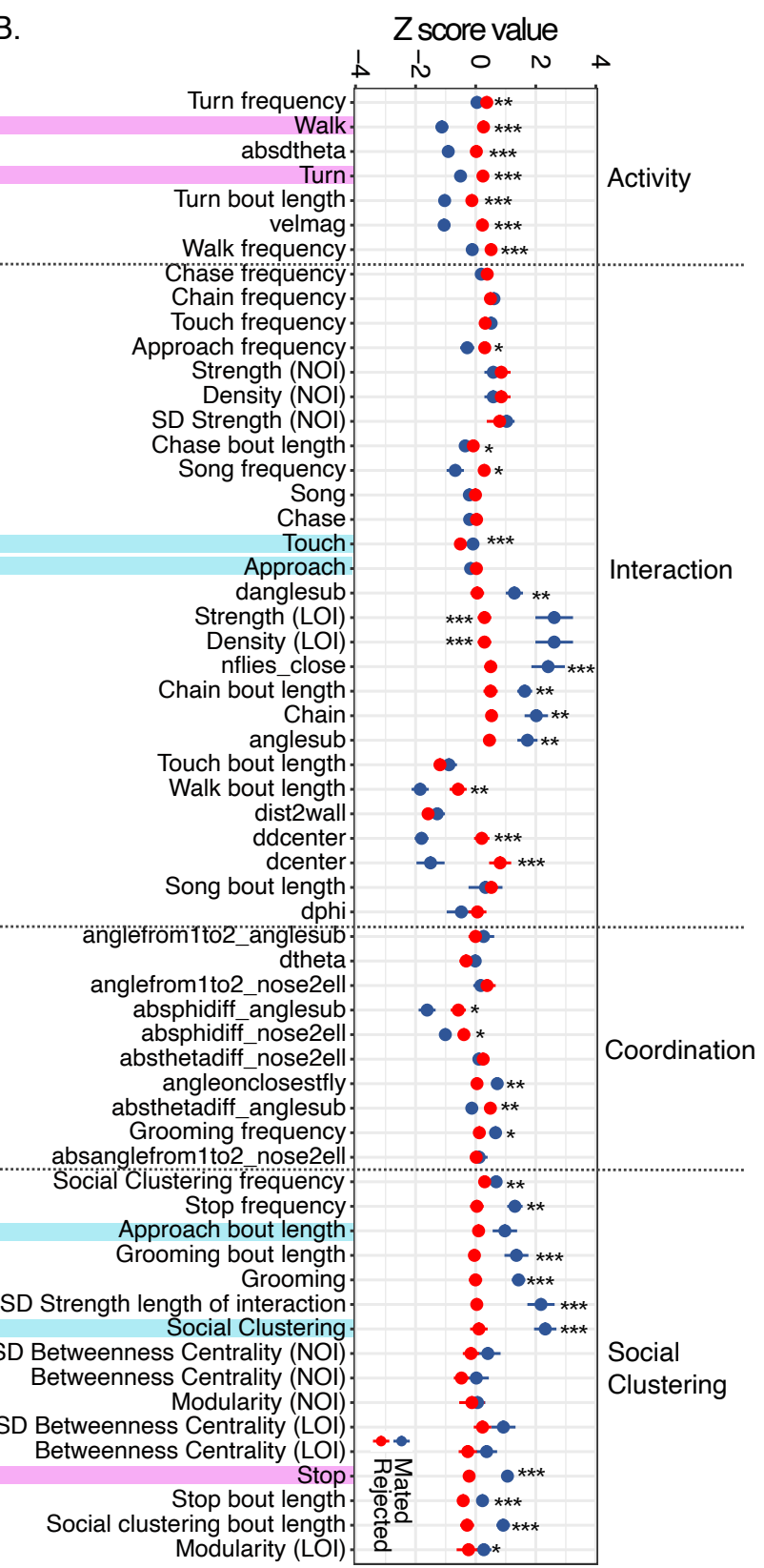

Figure 1. Failure to mate modifies action selection upon encounters with rival male flies. A. Schematic representation of the behavioral paradigm. B. Behavioral signatures of mated versus rejected WT male flies. Data is represented as normalized $\mathrm{Z}$ scores of 60 behavioral parameters, $n=18$. Statistical significance was determined by t-test for normally distributed parameters or Wilcoxon test for non-normally distributed parameters. LOI: calculated according to the length of interactions. NOI: calculated according to the number of interactions. Features mentioned in the results section are highlighted in pink and blue. C. Average number of flies close to any fly (threshold $\leq 1.5$ body length) along the experiment. 
A.

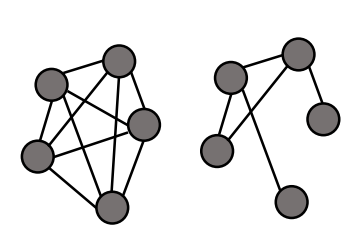

High Density Low Density
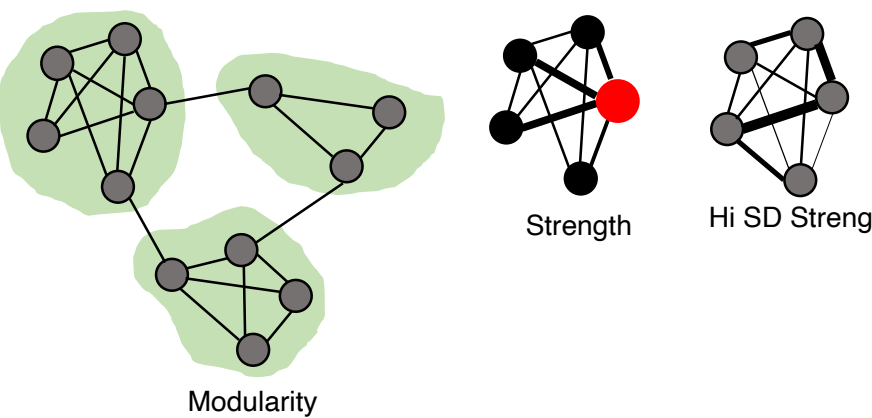

Hi SD Strength Low SD Strength

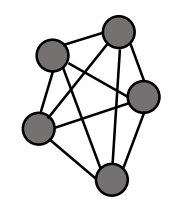

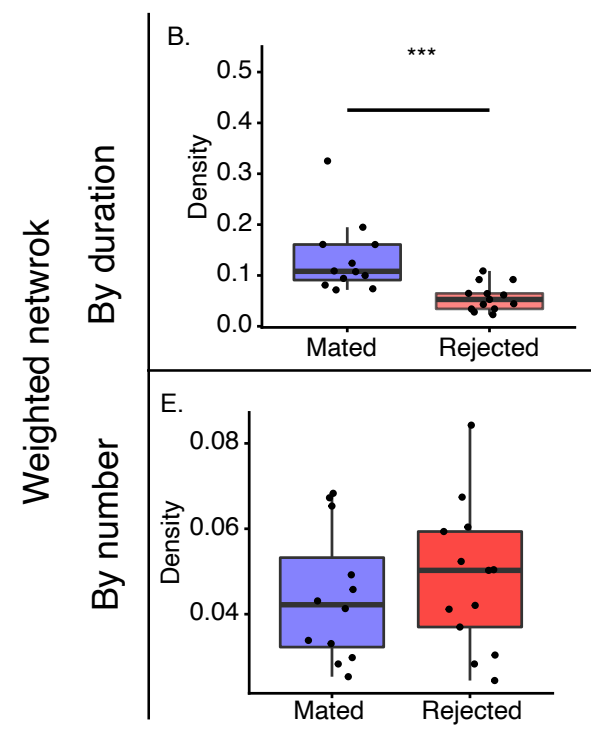
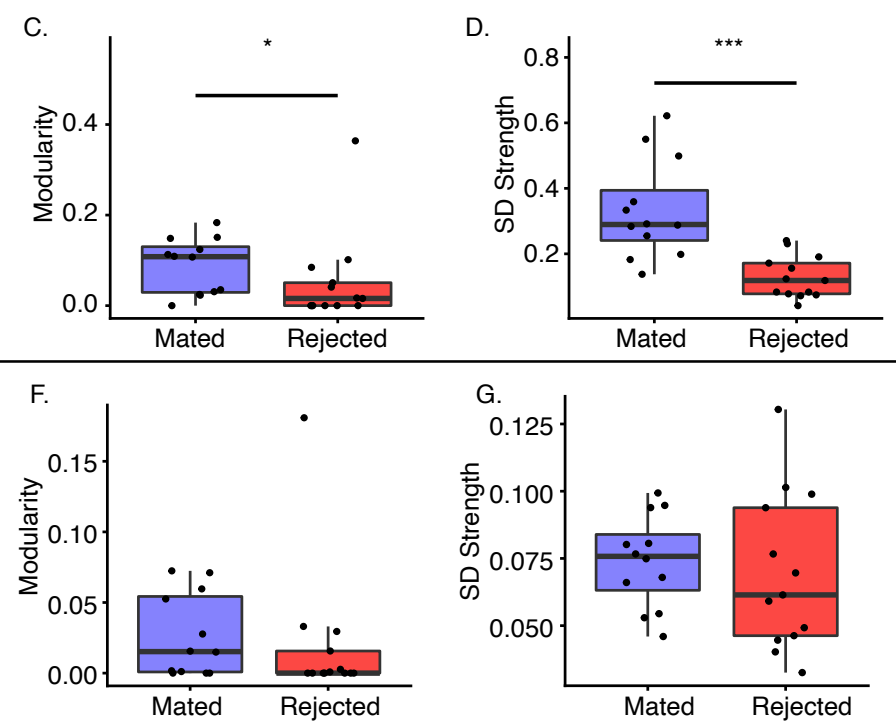

H.

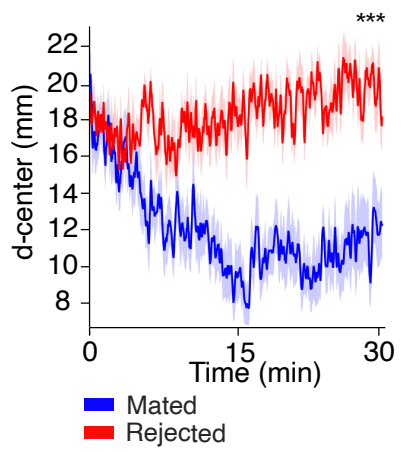

I.

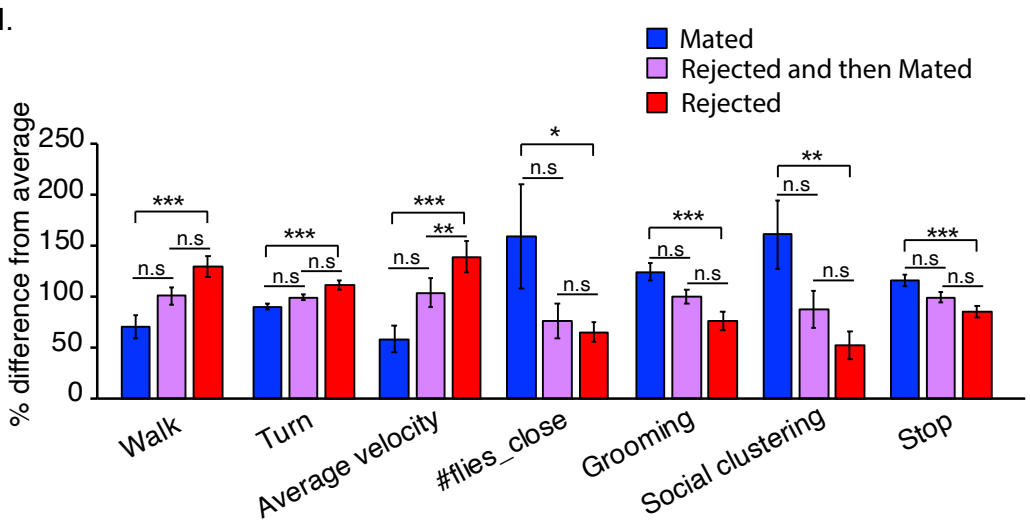

Figure 2. Sexual deprivation promotes social avoidance. A. Illustration of network parameters. Density of networks represents how saturated they are compared to the maximum possible. Modularity is a measure of the division of a network into sub-networks. Strength is proportional to vertex size (high in red individual). Standard deviation (SD) strength is a measure of the heterogeneity of the connections between individuals. B-G. Social network analysis of groups composed of rejected (red) and mated (blue) male flies. Network density, modularity, and SD strength calculated by network weights according to duration (A-C) or number of interactions (D-F), $\mathrm{n}=$ 18. Statistical significance was determined by Wilcoxon test and FDR correction for multiple tests, ${ }^{*} p<0.05,{ }^{* *} p$ $<0.01, * * * p<0.001$. Error bars signify SEM. H. Rejected male flies maintain large distances between flies along time, $\mathrm{n}=18$ Statistical significance was determined by Wilcoxon test. Data is presented as mean \pm SEM. I . Rejected and then mated male flies depict intermediate levels of activity and social interaction features when compared to rejected or mated cohorts. $\mathrm{n}=8$. Statistical significance was determined by Wilcoxon test and FDR correction for multiple tests, ${ }^{*} p<0.05,{ }^{* *} p<0.01,{ }^{* * *} p<0.001$. Error bars signify SEM. 
A.

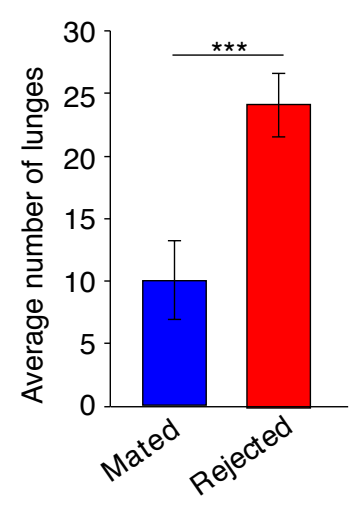

B.

C.
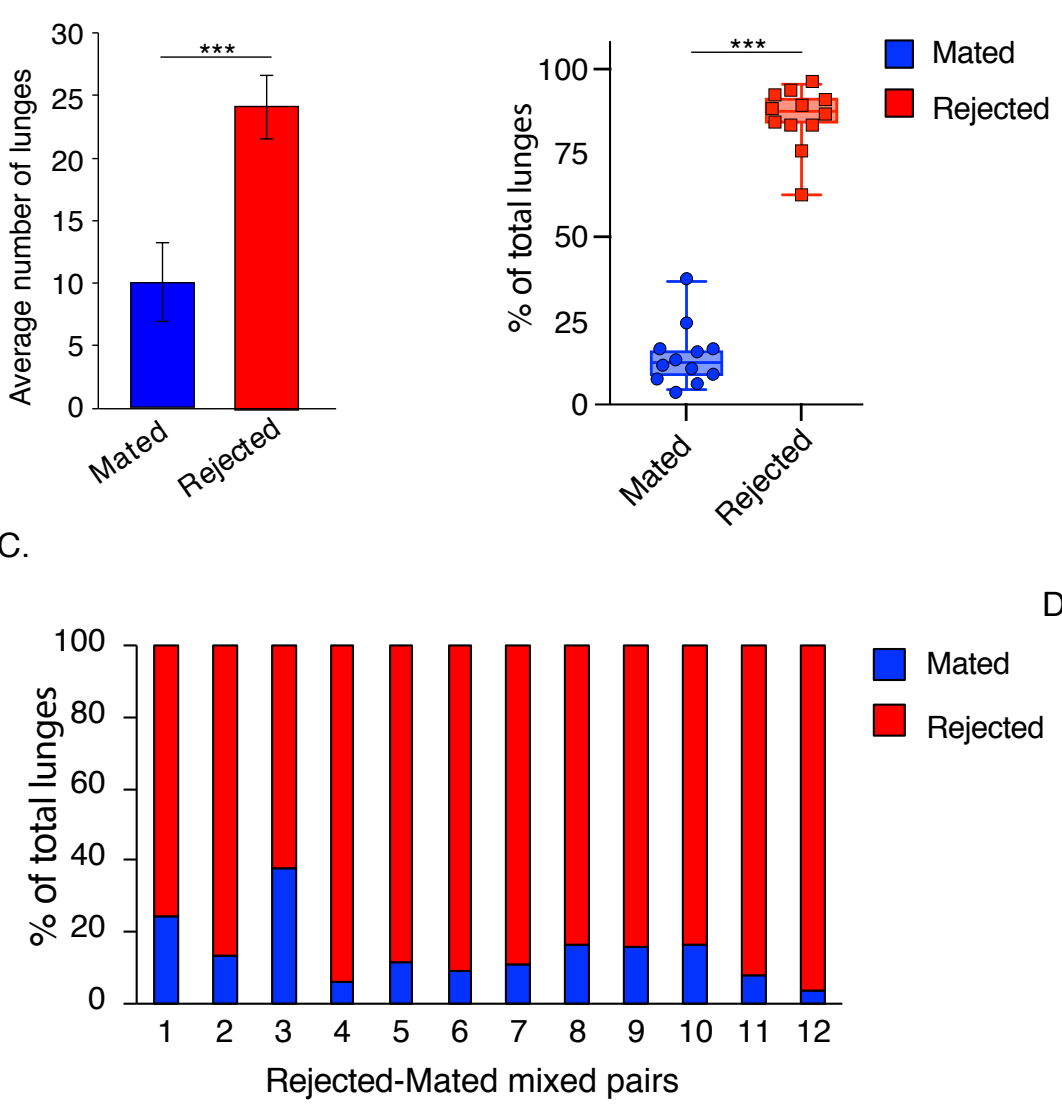

D.

Figure 3. Sexual deprivation modulates competitive behaviors. A.-C. Aggression display (number of lunges) was compared between pairs of rejected and mated male flies $(n=16$, statistical significance determined by T-test, $p<0.005$ (A), and mixed pairs $(\mathrm{n}=12)(\mathbf{B}-\mathbf{C})$. The $\log 2$ ratio between the number of lunges in rejected and mated flies was calculated for each pair, and then a one-sample T-test was performed to test whether the mean ratio was significantly different than $0, p<0.005$. Data is presented as the mean $\pm \mathrm{SEM}$. D. Duration of copulation in rejected vs. naïve male flies. Statistical significance was determined by T-test, $p<0.001$. Data is presented as mean $\pm \mathrm{SEM}$, $\mathrm{n}=25$. 
A.

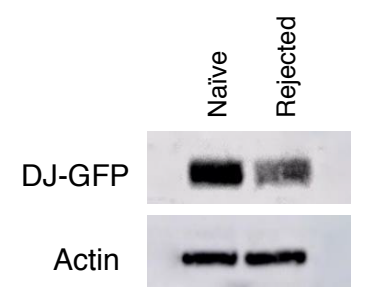

B.

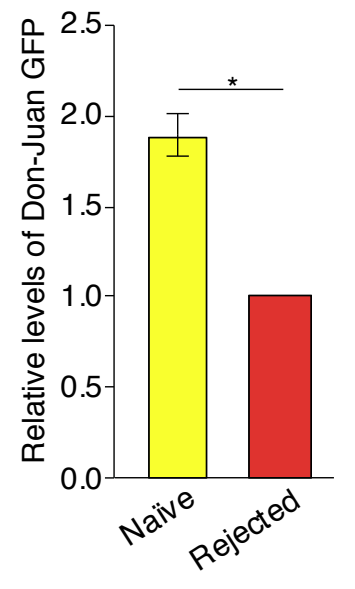

C.

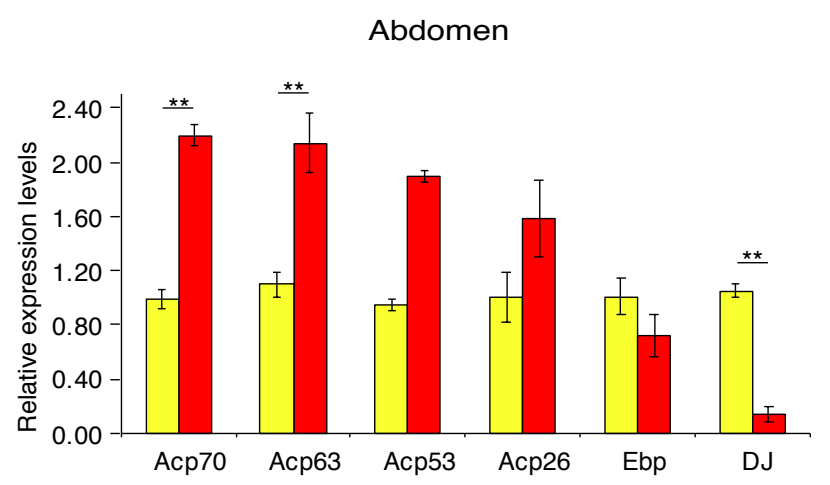

D.

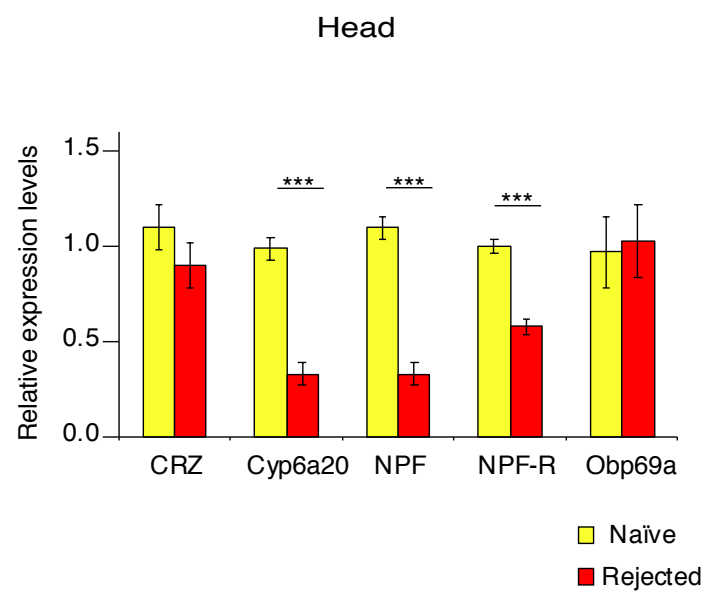

Figure 4. Failure to mate modulate sperm and seminal fluid composition. A,B. Protein lysates prepared from abdomen of rejected and naïve male flies and were analyzed for the relative levels of Don-Juan-GFP using western blot, actin was used as a loading control. Expression levels of Don-Juan-GFP protein were quantified and normalized to actin levels $(\mathrm{n}=3)$, Statistical significance was determined by T-test, $p<0.05$ (F). C.D. Relative transcript levels of candidate genes expressed in abdomen $(\mathrm{G})$ and heads $(\mathrm{H})$ of rejected and naïve male flies were quantified by qRT-PCR, $\mathrm{n}=6$ independent experiments of 15-20 fly heads and abdomen. Statistical significance 0.005 . 
A.

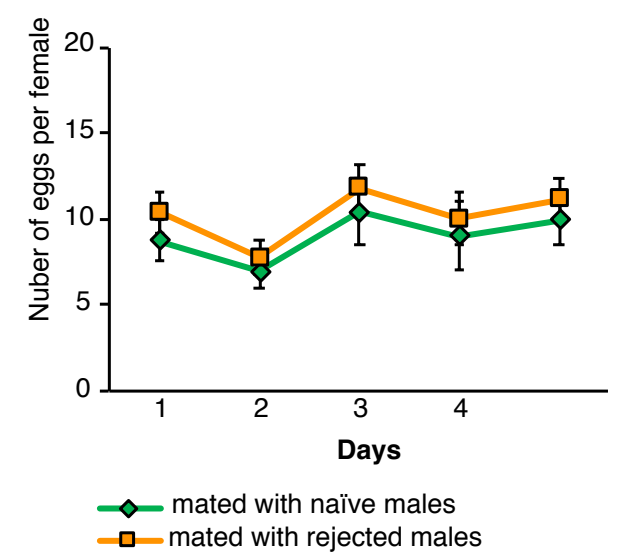

C.

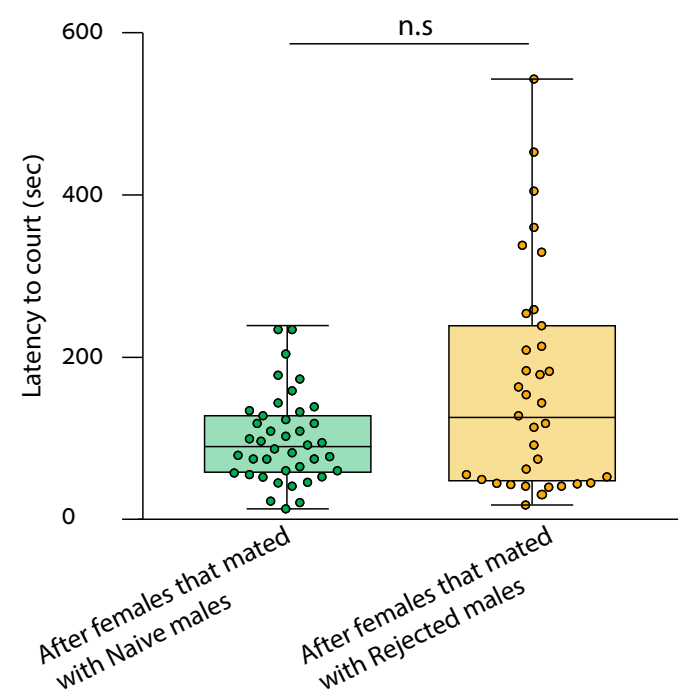

B.

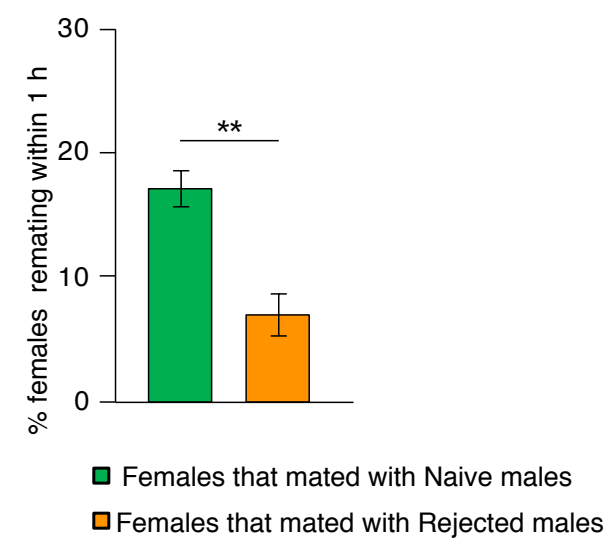

D.

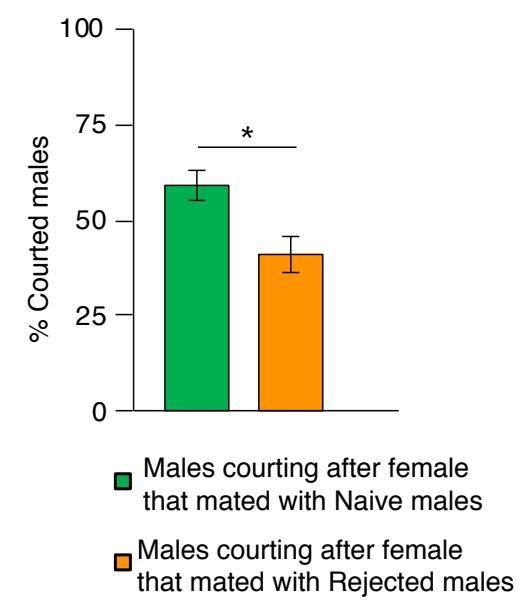

587 Figure 5. Effect of male rejection on female's fertility and remating tendencies. A. Number of eggs laid by 588 females that copulated with rejected or naïve male flies over the course of 5 days. Statistical significance was determined using two-way ANOVA repeated measure, $\mathrm{n}=28 p>0.05$. B. Female receptivity to re-mate with male flies $24 \mathrm{~h}$ after the first mating with rejected or naïve male flies was scored bycounting the precent of female flies that mated during 1 hour of test. Data is presented as the mean \pm SEM, $n=4$ repeats. Statistical significance was determined by Cochran-Mantel-Haenszel Chi-square test, $p<0.005$. C. Mean courtship latencies of rejected or naïve male flies towards mated female flies ( 24 hours post first mating), $n=25$. Statistical significance was determined by Mann-Whitney U-test, N.S., $p>0.05$. D. Number of new males that courted females that were previously mated with rejected or naïve male flies, $\mathrm{n}=25$. Statistical significance was determined by $\mathrm{T}$-test, $p<$ 0.05 . 
bioRxiv preprint doi: https://doi.org/10.1101/2021.04.27.441612; this version posted April 28, 2021. The copyright holder for this preprint (which was not certified by peer review) is the author/funder, who has granted bioRxiv a license to display the preprint in perpetuity. It is made available under aCC-BY-NC-ND 4.0 International license.

\begin{tabular}{|c|c|c|c|}
\hline Definition & Description & Definition & Description \\
\hline \multirow{2}{*}{ dnose2ell } & \multirow{2}{*}{$\begin{array}{l}\text { Minimum distance from any point of this } \\
\text { animal nose to the ellipse of other flies. }\end{array}$} & Walk & Fly moves. \\
\hline & & Stop & Fly is still. \\
\hline \multirow{3}{*}{$\begin{array}{l}\text { absanglefrom1to2 } \\
\text { nose2ell }\end{array}$} & \multirow{3}{*}{$\begin{array}{l}\text { Absolute difference between direction to } \\
\text { closest animal based on dnose2ell and } \\
\text { current animal's orientation (rad). }\end{array}$} & Turn & Changes in fly's direction. \\
\hline & & Touch & Fly actively touches another fly. \\
\hline & & \multirow{2}{*}{ Approach } & \multirow{2}{*}{$\begin{array}{l}\text { Fly approaches another fly and perform } \\
\text { interaction (active or passive). }\end{array}$} \\
\hline absdtheta & Angular speed (rad/s). & & \\
\hline \multirow{3}{*}{$\begin{array}{l}\text { absphidiff } \\
\text { anglesub }\end{array}$} & \multirow{3}{*}{$\begin{array}{l}\text { Absolute difference in velocity direction } \\
\text { between current animal and closest } \\
\text { animal based on anglesub (rad). }\end{array}$} & Aggregation & Fly sits in a group of 3 or more flies. \\
\hline & & Grooming & Fly grooms. \\
\hline & & Chase & Fly chases another fly. \\
\hline \multirow{3}{*}{$\begin{array}{l}\text { absphidiff } \\
\text { nose2ell }\end{array}$} & \multirow{3}{*}{$\begin{array}{l}\text { Absolute difference in velocity direction } \\
\text { between current animal and closest } \\
\text { animal based on dnose2ell (rad). }\end{array}$} & Chain & Chase with 3 or more flies. \\
\hline & & Song & Fly moves one wing next to another fly. \\
\hline & & $\begin{array}{l}\text { Behavior bout } \\
\text { length }\end{array}$ & $\begin{array}{l}\text { Length of the longest sequence of frames in } \\
\text { which the behavior occurred per fly. }\end{array}$ \\
\hline $\begin{array}{l}\text { absthetadiff } \\
\text { anglesub }\end{array}$ & $\begin{array}{l}\text { Absolute difference in orientation } \\
\text { between current animal and closest } \\
\text { animal based on anglesub (rad). }\end{array}$ & Behavior frequency & $\begin{array}{l}\text { Length of the movie minus the length of the } \\
\text { longest sequence of frames in which the } \\
\text { behavior didn't occurred for each fly. }\end{array}$ \\
\hline $\begin{array}{l}\text { absthetadiff } \\
\text { nose2ell }\end{array}$ & $\begin{array}{l}\text { Absolute difference in orientation } \\
\text { between this animal and closest animal } \\
\text { based on dnose2ell (rad). }\end{array}$ & $\begin{array}{l}\text { Density SD by length } \\
\text { of interactions (LOI) }\end{array}$ & $\begin{array}{l}\text { Accumulated interactions' length relative to } \\
\text { the maximum interactions' length possible. }\end{array}$ \\
\hline $\begin{array}{l}\text { anglefrom1to2 } \\
\text { anglesub }\end{array}$ & $\begin{array}{l}\text { Angle to closest (based on angle } \\
\text { subtended) animal's centroid in current } \\
\text { animal's coordinate system (rad). }\end{array}$ & $\begin{array}{l}\text { Modularity by } \\
\text { length of } \\
\text { interactions (LOI) }\end{array}$ & $\begin{array}{l}\text { Representation of how much the network is } \\
\text { divided into modules according to } \\
\text { interactions' length. }\end{array}$ \\
\hline \multirow{2}{*}{$\begin{array}{l}\text { anglefrom1to2 } \\
\text { nose2ell }\end{array}$} & \multirow{2}{*}{$\begin{array}{l}\text { Angle to closest (based on distance from } \\
\text { nose to ellipse) animal's centroid in } \\
\text { current animal's coordinate system (rad). }\end{array}$} & $\begin{array}{l}\text { Strength by length } \\
\text { of interactions (LOI) }\end{array}$ & Length of interactions of a certain fly. \\
\hline & & \multirow{2}{*}{$\begin{array}{l}\text { SD Strength } \\
\text { according to length } \\
\text { of interactions (LOI) }\end{array}$} & \multirow{2}{*}{$\begin{array}{l}\text { Standard deviation of the strengths according } \\
\text { to interactions' length of flies from the same } \\
\text { movie. }\end{array}$} \\
\hline \multirow[b]{2}{*}{ angleonclosestfly } & \multirow{2}{*}{$\begin{array}{l}\text { Angle of the current animal's centroid in } \\
\text { the closest (based on distance from nose } \\
\text { to ellipse) animal's coordinate system } \\
\text { (rad). }\end{array}$} & & \\
\hline & & $\begin{array}{l}\text { Betweenness } \\
\text { Centrality by length } \\
\text { of interactions (LOI) }\end{array}$ & $\begin{array}{l}\text { A measure of centrality of a certain fly based } \\
\text { on shortest paths according to interactions' } \\
\text { length. }\end{array}$ \\
\hline anglesub & $\begin{array}{l}\text { Maximum total angle of animal's field of } \\
\text { view (fov) occluded by another animal } \\
\text { (rad). }\end{array}$ & $\begin{array}{l}\text { SD Betweenness } \\
\text { Centrality by length } \\
\text { of interactions (LOI) }\end{array}$ & $\begin{array}{l}\text { Standard deviation of the betweenness } \\
\text { centralities according to interactions' length } \\
\text { of flies from the same movie. }\end{array}$ \\
\hline danglesub & $\begin{array}{l}\text { Change in maximum total angle of } \\
\text { animal's view occluded by another animal } \\
(\mathrm{rad} / \mathrm{s}) \text {. }\end{array}$ & $\begin{array}{l}\text { Density by number } \\
\text { of interactions (NOI) }\end{array}$ & $\begin{array}{l}\text { Interactions' number relative to the maximum } \\
\text { interactions' number possible. }\end{array}$ \\
\hline dcenter & $\begin{array}{l}\text { Minimum distance from this animal's } \\
\text { center to other animal's center (mm). }\end{array}$ & $\begin{array}{l}\text { Modularity Strength } \\
\text { by number of } \\
\text { interactions (NOI) }\end{array}$ & $\begin{array}{l}\text { Representation of how much the network is } \\
\text { divided into modules according to } \\
\text { interactions' number. }\end{array}$ \\
\hline \multirow[t]{2}{*}{ ddcenter } & \multirow[t]{2}{*}{$\begin{array}{l}\text { Change in minimum distance between } \\
\text { this animal's center and other flies' } \\
\text { centers }(\mathrm{mm} / \mathrm{s}) \text {. }\end{array}$} & $\begin{array}{l}\text { Strength by number } \\
\text { of interactions (NOI) }\end{array}$ & Number of interactions of a certain fly. \\
\hline & & \multirow{2}{*}{$\begin{array}{l}\text { SD Strength by } \\
\text { number of } \\
\text { interactions (NOI) }\end{array}$} & \multirow{2}{*}{$\begin{array}{l}\text { Standard deviation of the strengths according } \\
\text { to interactions' number of flies from the same } \\
\text { movie. }\end{array}$} \\
\hline dist2wall & $\begin{array}{l}\text { Distance to the arena wall from the } \\
\text { animal's center }(\mathrm{mm}) \text {. }\end{array}$ & & \\
\hline dphi & Change in the velocity direction (rad/s). & \multirow{2}{*}{$\begin{array}{l}\text { Betweenness } \\
\text { Centrality by } \\
\text { number of } \\
\text { interactions (NOI) }\end{array}$} & \multirow{2}{*}{$\begin{array}{l}\text { A measure of centrality of a certain fly based } \\
\text { on shortest paths according to interactions' } \\
\text { number. }\end{array}$} \\
\hline dtheta & Angular velocity (rad/s). & & \\
\hline nflies_close & $\begin{array}{l}\text { Number of flies within } 2 \text { body lengths } \\
(4 a) \text {. }\end{array}$ & \multirow[b]{2}{*}{$\begin{array}{l}\text { SD Betweenness } \\
\text { centrality (by } \\
\text { number of } \\
\text { interactions (NOI) }\end{array}$} & \multirow[b]{2}{*}{$\begin{array}{l}\text { Variance of the betweenness centralities } \\
\text { according to interactions' number of flies from } \\
\text { the same movie. }\end{array}$} \\
\hline velmag & Speed of the center of rotation $(\mathrm{mm} / \mathrm{s})$. & & \\
\hline
\end{tabular}


A.

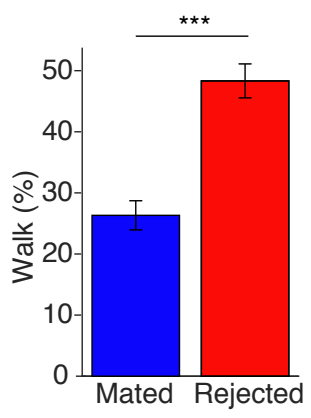

D.

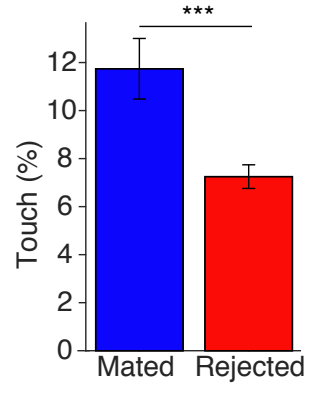

G.

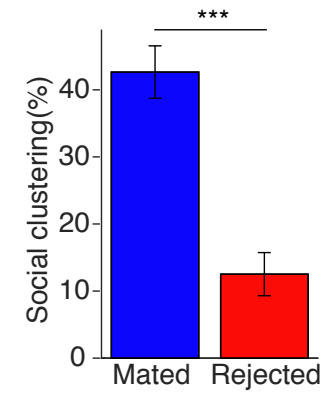

B.

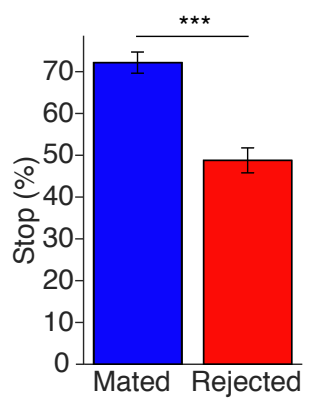

E.

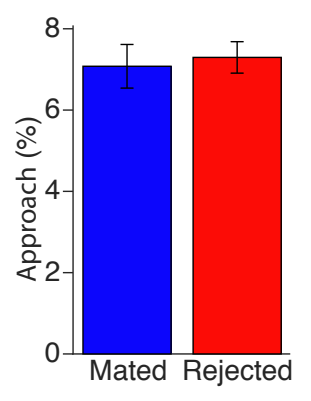

C.

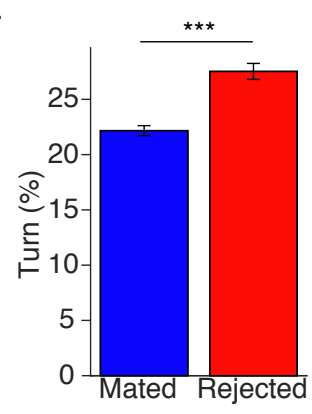

F.

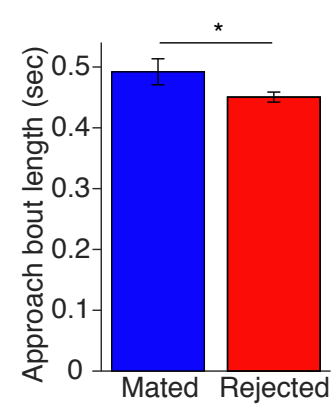

598 Supplementary Figure 1. Behavior classifier analysis depicts mean values of the behaviors averaged across the 599 experiment: walking (A), Stop (B) body turns (C), close touch behavior (D), approach, bout duration of approach 600 behavior $(F)$ and social aggregation $(G) . n=18 \mathrm{t}$ test for normally distributed parameters or Wilcoxon test for non601 normally distributed parameters. 
bioRxiv preprint doi: https://doi.org/10.1101/2021.04.27.441612; this version posted April 28, 2021. The copyright holder for this preprint (which was not certified by peer review) is the author/funder, who has granted bioRxiv a license to display the preprint in perpetuity. It is made available under aCC-BY-NC-ND 4.0 International license.

602

603

604

605
Number of housed male flies

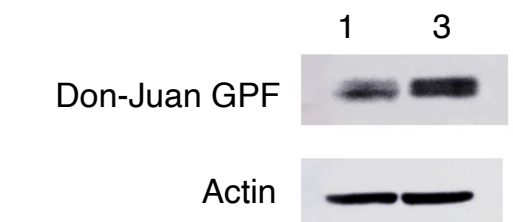

Actin

\section{Supplementary Figure 2. The expression of Don-Juan protein in sensitive to the presence of rival male flies.} Relative expression levels of Don-Juan-GFP in male flies in single or grouped housed male flies. 